\title{
Las concepciones aristotélicas de la vida buena y la falacia naturalista
}

\author{
Rocío CÁZARES BLANCO \\ Unidad Académica de Filosofía \\ Universidad Autónoma de Zacatecas \\ caz.rocio@gmail.com
}

\begin{abstract}
Resumen: Las concepciones de la vida buena de corte aristotélico frecuentemente son criticadas desde diversas posiciones éticas, como el antinaturalismo de G.E. Moore o los subjetivismos emotivistas y prescriptivistas, porque supuestamente cometen la falacia naturalista en su planteamiento acerca de cómo han de evaluarse moralmente las características, acciones, intenciones y facultades de los seres humanos. En este artículo, luego de examinar y desechar la estrategia de Alfonso Gómez-Lobo para rechazar esa acusación, propongo otra estrategia y sostengo que las concepciones aristotélicas inclusivistas de la vida buena pueden librarse de tal crítica apelando al concepto de "hechos brutos relativamente a..." propuesta por G.E. Anscombe.
\end{abstract}

Palabras clave: Aristóteles, vida buena, falacia naturalista, hechos brutos

\begin{abstract}
Aristotelian conceptions of the good life are often criticized from several ethical positions, like G.E. Moore's anti-naturalism or emotivism and prescriptivism. Aristotelians are accused of committing the naturalistic fallacy when they make a moral evaluation of features, actions, intentions and faculties of human beings. In this paper, I examine and refute Alfonso GómezLobo's strategy to reject that accusation; then, I propose another strategy and I argue that inclusivist Aristotelian conceptions of the good life could be freed from such criticism if they appeal to the concept of "brute facts relative to" as proposed by G.E. Anscombe.
\end{abstract}

Key words: Aristotle, good life, naturalistic fallacy, brute facts

Frecuentemente se objeta que las concepciones de la vida buena de corte aristotélico son filosóficamente insostenibles porque cometen la llamada "falacia naturalista", es decir, pretenden sacar conclusiones evaluativas sobre la vida buena a partir de premisas fácticas acerca de la naturaleza humana. En este artículo argumento a favor de una vía para sostener una concepción de la vida buena de corte aristotélico sin dar un paso injustificado del es al debe. Conviene precisar que no es mi intención hacer un trabajo de interpretación textual de la Ética nicomáquea (en adelante $E N$ ) ni sugerir que la vía de defensa que propongo pueda atribuirse a Aristóteles; pretendo demostrar, únicamente, que tal vía funciona para salvar de la crítica de la falacia naturalista a una concepción de la vida buena que cabe reconocer como de corte 
aristotélico y que es afín, específicamente, a una interpretación inclusivista del eudemonismo aristotélico. Me concentro en la defensa de una concepción de la vida buena de este tipo porque me parece más verosímil que una concepción afín a la interpretación intelectualista; determinar si la estrategia de defensa que propongo funciona también en este último caso es algo que escapa a los objetivos de este artículo.

En la sección 1 haré un breve análisis de la concepción de la eudaimonia en Aristóteles para mostrar que, en efecto, él parece partir de descripciones acerca de las actividades y funciones humanas para justificar conclusiones normativas acerca de lo que constituye una vida buena o eudaímōn. En la sección 2 revisaré la defensa que hace Alfonso Gómez-Lobo $(1991,1989)$ de la concepción aristotélica de la eudaimonia, contra la acusación de que comete la falacia naturalista; sostendré que, aunque ciertamente el examen de Gómez-Lobo aporta claridad a la comprensión de dicha concepción, es insuficiente para desechar la crítica de que supone un paso injustificado del es al debe. En la sección 3 examinaré una perspectiva neoaristotélica acerca de las evaluaciones de "bondad" en el caso de los seres vivos, incluyendo por supuesto a los humanos, y para ello tendré en cuenta las propuestas de Philippa Foot (2001) y Alasdair MacIntyre (2001). Por último, en la sección 4 intentaré defender un argumento de corte aristotélico acerca de lo que es una vida buena para un ser humano, consistente con la perspectiva neoaristotélica examinada en la sección anterior y afín a una interpretación inclusivista del eudemonismo aristotélico; utilizando el concepto de "ser un hecho bruto relativamente a...", introducido por G.E. Anscombe (1958a, 1958b), sostendré que dicho argumento es inmune a la crítica de la falacia naturalista.

Mi conclusión general es que resulta posible defender una concepción inclusivista de la vida buena de corte aristotélico que no dé lugar a la crítica de que se comente la falacia naturalista, siempre y cuando la relación entre determinados hechos acerca de la naturaleza humana y determinadas evaluaciones acerca de la vida buena se explique haciendo uso del concepto de "ser un hecho bruto relativamente a...", introducido por G.E. Anscombe.

\section{El naturalismo ético de Aristóteles y la falacia naturalista}

En la EN Aristóteles defiende una concepción de la eudaimonia o de la vida buena que cabe calificar como objetivista y naturalista porque, de acuerdo con él, aquello en lo que consiste tener una vida buena 
depende de las características que las personas tenemos por el hecho de ser humanas. Así, lo que sea una vida buena no está determinado por alguna voluntad trascendente y tampoco por los deseos, preferencias o gustos personales de los agentes (los cuales pueden variar de un individuo a otro y no dependen, en principio, de cuáles sean las fuentes de sus creencias y de sus experiencias), sino que está determinado por las características de la naturaleza humana, la cual, por supuesto, es la misma para todas las personas.

¿Cuáles son las características de la naturaleza humana? Para responder a esto, Aristóteles hace un análisis de los diversos tipos de actividades o funciones propias de la vida de los humanos y encuentra que éstas son de dos tipos: las actividades o funciones que podemos llamar "corporales", como nutrirnos, crecer, sentir y tener impulsos y apetitos; y las que involucran el pensamiento, que incluyen el razonamiento teórico y el razonamiento práctico, es decir, el razonamiento aplicado tanto a la búsqueda de la verdad como a la dirección que hayan de tomar nuestras acciones. ${ }^{1}$ Las actividades de nutrición y crecimiento, sostiene Aristóteles, son suficientes para caracterizar la naturaleza de los vegetales; se tienen que agregar las actividades de la sensación, los apetitos y los impulsos para caracterizar la naturaleza de los animales no humanos; y por último, además de todo lo anterior, hay que añadir las actividades del pensamiento teórico y práctico para tener una descripción completa de las actividades y funciones de los humanos. Aristóteles presenta este último tipo de funciones como el distintivo de los humanos.

¿Cómo a partir de la descripción de la naturaleza humana Aristóteles pretende derivar una concepción de la eudaimonia o de la vida buena? Entre los estudiosos encontramos dos tipos de interpretaciones sobre la concepción aristotélica de la eudaimonia: las inclusivistas o comprehensivas y las exclusivistas o intelectualistas. ${ }^{2}$ Estas últimas se caracterizan por considerar que la eudaimonia consiste, ya sea de manera exclusiva o predominante, en el ejercicio excelente del pensamiento teórico,

${ }^{1}$ Cfr. Aristóteles, EN 1097b30-1098a5, 1102a25-1103a5, 1139a1-36; Gómez Robledo 1957, p. 23.

${ }^{2}$ Entre los especialistas que han defendido interpretaciones inclusivistas o comprehensivas de la teoría aristotélica de la eudaimonia están Cooper 1986, pp. 89115; Ackrill 1980, pp. 15-33; Rorty 1980, pp. 377-394; Irwin 1993, pp. 211-250; y Urmson 1988, pp. 118-127. En cambio, entre quienes han argumentado en algunos de sus textos a favor de interpretaciones exclusivistas o intelectualistas de la teoría aristotélica de la eudaimonia están Gómez Robledo 1957, pp. 131-140; y Kenny 1965-1966, pp. 93-102. 
mientras que las primeras entienden la eudaimonia como la posesión de un conjunto de bienes que puede ser más o menos amplio, pero incluye siempre el ejercicio excelente del pensamiento práctico y teórico. En lo que sigue me concentraré en una interpretación ampliamente inclusiva, porque me parece la más verosímil para explicar qué es una vida buena o eudaímōn; además, son versiones de ésta las que han defendido neoaristotélicos como Philippa Foot (2001) y Alasdair MacIntyre (2001), cuyas propuestas consideraré en la sección 3 de este artículo. Según esta interpretación, una persona que tiene una vida eudaímōn es la que disfruta de todos los tipos de cosas que son en sí mismas un bien para los humanos y lo que cabe ser reconocido en sí mismo como un bien para los humanos depende, precisamente, de las características de la naturaleza humana. ${ }^{3}$

Uno de los pasajes más citados en apoyo de la interpretación inclusivista de la eudaimonia es EN 1097b16-20, aunque, como muestra el análisis de Gavin Lawrence, ${ }^{4}$ es un pasaje polémico porque admite la siguiente doble lectura:

(A) la eudaimonia es lo más digno de ser elegido con tal de que no se le agregue nada - lo cual se puede hacer, y si se hiciera, entonces por supuesto que la adición de cualquier otro bien por pequeño que sea produciría un bien mayor y, por ello, más digno de ser elegido. [...]

(NA) la eudaimonia es lo más digno de ser elegido con tal de que no se le agregue nada - lo cual no se puede hacer, puesto que si se pudiera, entonces por supuesto que la adición de cualquier otro bien por pequeño que sea produciría un bien más grande y, por ello, más digno de ser elegido. ${ }^{5}$

De acuerdo con (A), la eudaimonia se concibe como un bien particular entre otros bienes particulares, como son la salud, la amistad, la valentía, el placer, etcétera. Así, aunque al considerar individualmente

${ }^{3}$ No pretendo sostener que la $E N$ ofrezca mayor evidencia para atribuirle a Aristóteles una concepción de la eudaimonia inclusivista que una concepción intelectualista, ni que permita fundamentar mejor un inclusivismo amplio que uno menos abarcante; como dije antes, mi intención no es hacer un trabajo de interpretación textual. Para los propósitos de este artículo me basta caracterizar la concepción de la eudaimonia ampliamente inclusiva a la que me he referido y que en lo sucesivo llamaré simplemente "interpretación inclusivista", la cual es una de las posibles interpretaciones del eudemonismo aristotélico.

${ }^{4}$ Cfr. Lawrence 1997, pp. 32-37.

${ }^{5}$ Ibid., p. 34; la traducción es mía. 
los distintos bienes existentes la eudaimonia resulte ser el más deseable, se obtiene un bien mayor con la adición de cualquier otro bien. La interpretación intelectualista de la eudaimonia ${ }^{6}$ es consistente con la lectura (A) de EN 1097b16-20 porque, si el mayor bien que puede perseguir un ser humano es la excelencia en la actividad del pensamiento teórico, entonces, una vida eudaímōn será aquélla en la que se ejercita con excelencia dicha actividad; sin embargo, será posible alcanzar un bien mayor que la eudaimonia si a ésta se le añade(n) otro(s) bien(es) particular(es).

En contraste con lo anterior, según (NA) el pasaje EN 1097b16-20 establece la imposibilidad de obtener un bien mayor que la eudaimonia mediante la adición de otro bien. Esto sólo puede ser el caso (argumentan algunos de los defensores de la interpretación inclusivista) ${ }^{7}$ si la eudaimonia no figura en la lista de bienes particulares, sino que se la concibe como el bien que los incluye a todos. Al conjunto formado por todos los bienes existentes no se le puede agregar ningún otro bien para producir un bien más deseable, puesto que no hay ningún otro que dicho conjunto no incluya.

Ahora bien, si apoyándose en (NA) se argumenta que Aristóteles concibe la eudaimonia constituida por el conjunto de todos los tipos de bienes, ¿qué es lo que incluye ese conjunto? Realizar con excelencia todas y cada una de las funciones o actividades propias de nuestra naturaleza, según la interpretación inclusivista de la eudaimonia, es en lo que consiste el bien para nosotros. Dado que la nutrición, el crecimiento, la sensibilidad, etc., son funciones propias (aunque no exclusivas) de los humanos, tener una buena digestión, no tener problemas hormonales, tener buena vista, etc., es en lo que consiste un primer tipo de bienes: los llamados "bienes del cuerpo". Y dado que las actividades del razonamiento teórico y práctico son distintivas de los humanos (y exclusivas de nosotros, según Aristóteles, lo que les confiere un valor superior respecto de otras funciones), realizar estas actividades con excelencia constituye un segundo tipo de bienes: los que llama "bienes del alma". Una persona posee tales bienes cuando, por un lado, usa su razonamiento teórico para - por ejemplo- comprender una teoría astronómica o encontrar la solución a un problema matemático y, por otro lado, usa su razonamiento práctico para — por ejemplo- decidir por cuál partido político ha de votar, qué hacer ante un régimen tiránico o cómo organizar las actividades que realizará durante el día.

${ }^{6}$ Interpretación que encuentra apoyo sobre todo en el Libro $\mathrm{X}$ de $E N$; véanse, por ejemplo, 1177a12-25 y 1177b15-1178a10.

${ }^{7}$ Cfr. Irwin 1993, pp. 213-214; Ackrill 1980, pp. 20-21.

Diánoia, vol. LV, no. 65 (Noviembre 2010). 
Aristóteles considera, además, que aun la obtención de los bienes del cuerpo requiere del buen uso de la razón práctica, de la virtud de la phronesis.

Nótese que muchos de los bienes del cuerpo y del alma no se pueden alcanzar en la ausencia de algunas cosas externas; por ejemplo, no es posible estar bien nutrido cuando se sufre de pobreza extrema y no se recibe apoyo alguno de otras personas o de las instituciones sociales. Es por esto que Aristóteles afirma que hay un tercer tipo de bienes: los que llama "bienes externos", como el alimento, la influencia política, la amistad, etc., que son instrumentalmente valiosos y, algunos de ellos, poseen además un valor intrínseco, como es el caso de la amistad. ${ }^{8}$ Son los bienes externos intrínsecamente valiosos, esos sin los cuáles dice Aristóteles - se ve deslucida o empañada nuestra dicha, ${ }^{9}$ los que forman parte del conjunto de bienes que constituyen la eudaimonia; siendo ésta un bien intrínsecamente valioso —además de ser el único bien que se persigue siempre por sí mismo y nunca como un medio para otra cosa, pues es el bien supremo- ${ }^{10}$ no puede incluir bienes cuyo valor es exclusivamente instrumental como partes constitutivas. Poseer algunos bienes externos que tienen un valor exclusivamente instrumental es condición necesaria para alcanzar algunos de los bienes corporales y del alma que forman parte de la eudaimonia, como hemos dicho, pero aquéllos no son parte constitutiva de ésta, mientras que los bienes externos con valor intrínseco son tanto condición necesaria como parte constitutiva de la eudaimonia.

Si la caracterización de la naturaleza humana permite identificar tres tipos de bienes intrínsecos (los del cuerpo, los del alma y algunos externos) porque tenerlos es en lo que consiste realizar con excelencia las funciones o actividades propias de nuestra naturaleza, y si la eudaimonia o la vida buena es aquella en la que se tienen todos los tipos de bienes existentes, entonces, se alcanza la eudaimonia cuando se tienen dichos bienes. ${ }^{11}$ Esta concepción de la vida buena pareciera, a primera vista, cometer la falacia naturalista, ya que a partir de premisas acerca de cómo es la naturaleza humana pretende sacar conclusiones acerca de lo que se necesita o se debe hacer para lograr una vida buena o eudaímōn; el argumento parecería tener la forma siguiente:

${ }^{8}$ Cfr. Aristóteles, EN 1099a24-b9.

${ }^{9}$ Cfr. Idem.

${ }^{10}$ Cfr. Ibid., 1097a25-b5.

${ }^{11}$ Cfr. Aristóteles, EN 1098b10-15; Urmson 1988, pp. 3, 21-22; Valdés 1991, pp. 70-71; Annas 1996, p. 248.

Diánoia, vol. LV, no. 65 (Noviembre 2010). 


\section{ARgumento 1}

(i) El conjunto de funciones o actividades $\varphi^{1}, \varphi^{2}, \varphi^{3} \ldots \varphi^{n}$ es característico de la naturaleza de los seres humanos.

Por lo tanto,

(ii) La eudaimonia o la vida buena consiste en realizar con excelencia $\varphi^{1}, \varphi^{2}, \varphi^{3} \ldots \varphi^{n}$.

La crítica de que Aristóteles comete una falacia al dar este paso del es al debe se puede plantear, con distintos matices, desde diversas posiciones éticas como el antinaturalismo de G.E. Moore o los subjetivismos de corte emotivista y prescriptivista; que sea adecuado o no atribuirle a Hume esta misma objeción a la concepción aristotélica de la eudaimonia es un asunto polémico, ${ }^{12}$ cuya discusión rebasa mis objetivos en este artículo. ¿Pueden los naturalismos aristotélicos responder a dicha crítica? Pienso que sí. Sin embargo, no todo rechazo de la crítica es igualmente efectivo. Antes de examinar otra respuesta que me parece más convincente, quiero revisar la respuesta que da Alfonso GómezLobo a la pregunta anterior.

\section{La respuesta de Alfonso Gómez-Lobo}

En sus artículos "The Ergon Inference" (1989) y "La fundamentación de la ética aristotélica" (1991), Alfonso Gómez-Lobo sostiene que los intérpretes de Aristóteles frecuentemente han fallado en identificar con precisión las premisas de su argumento sobre la eudaimonia y en algunos casos han malinterpretado términos y expresiones clave para dicho argumento, lo que ha dado lugar a que se plantee la crítica de la falacia naturalista. ${ }^{13}$ A través de un detallado trabajo de interpretación textual, Gómez-Lobo aporta evidencia a favor de que la correcta reconstrucción

\footnotetext{
${ }^{12}$ Alasdair MacIntyre está entre quienes han rechazado la interpretación estándar del famoso pasaje "es-debe" del Tratado de la naturaleza humana de Hume (cfr. p. 469 de la edición de L.A. Selby-Bigge), según la cual se comete una falacia siempre que se pretenden justificar juicios morales a partir de enunciados de hecho. Según MacIntyre, en ese pasaje Hume simplemente está criticando las explicaciones que otros han dado, en particular los moralistas religiosos, acerca de cuáles son los hechos relevantes para justificar juicios morales; estos hechos son, para Hume, necesidades, intereses y deseos humanos y no, como pretenden algunos, las leyes o propósitos divinos (cfr. MacIntyre 1969). Geoffrey Hunter también ha ofrecido otros argumentos en contra de la interpretación estándar del mencionado pasaje (cfr. Hunter 1969).

${ }^{13}$ Cfr. Gómez-Lobo 1989, pp. 170-172; Gómez-Lobo 1991, pp. 3-5.
} 
del argumento más general sobre la eudaimonia que se encuentra en la EN es la siguiente:

\section{Argumento 2}

(i) "Para todo $x$, si $x$ tiene un ergon $\varphi$, entonces $x$ será un buen $x$ si y sólo si produce buenas instancias de $\varphi$."

(ii) "El ergon del ser humano es actividad con logos."

De allí la conclusión:

(iii) "Un ser humano será un buen ser humano si y sólo si produce buenas instancias de actividad con logos". ${ }^{14}$

Como muestra el análisis textual de Gómez-Lobo, 2(ii) es una tesis fáctica que Aristóteles defiende en diversos pasajes de la $E N .{ }^{15}$ A diferencia de la premisa 1(i), mencionada al final de la sección anterior, 2(ii) se refiere a la función peculiar o característica de los humanos y no a todas las funciones o actividades que los seres humanos realizamos. Dependiendo de la amplitud con la que se caracterice el ergon o función peculiar de los humanos - esto es, si se le identifica con la actividad racional meramente teórica $\mathrm{o}$, además, con la práctica-, 2(ii) puede servir para defender una interpretación intelectualista o una interpretación inclusivista de la eudaimonia; en contraste, la premisa 1(i) forma parte de un argumento que sólo acepta una interpretación inclusivista de la eudaimonia.

La clave para evitar la crítica de la falacia naturalista es la premisa 2(i), la cual establece un criterio para valorar lo que es un buen $x$ cuando $x$ es algo que tiene un ergon distintivo. Una vez que se introduce una premisa evaluativa como 2(i) es posible, en principio, justificar la conclusión también evaluativa del argumento. Según Gómez-Lobo, Aristóteles parece considerar 2(i) como un principio evaluativo de sentido común obtenido por inducción enumerativa, ${ }^{16}$ ya que la observación de los diversos casos en que las personas juzgamos si una cosa $x$ es un buen $x$ o un mal $x$ nos permite concluir lo siguiente: para llevar a cabo

${ }^{14}$ Gómez-Lobo 1991, p. 13 [cito textualmente a Gómez-Lobo, modificando únicamente la notación de la numeración de las premisas y la relativa al ergon (él dice "un ergon y" y no "un ergon $\varphi$ ") por cuestiones de claridad en referencias posteriores a este artículo]. El mismo argumento se encuentra en Gómez-Lobo 1989, p. 182.

${ }^{15}$ Cfr. Gómez-Lobo 1989, pp. 176-182; Gómez-Lobo 1991, pp. 6-7 y 9-13.

${ }^{16}$ Cfr. Gómez-Lobo 1989, pp. 174-176 y 183; Gómez-Lobo 1991, p. 6. 
tales valoraciones empezamos por identificar la actividad o función $\varphi$ que es propia de la clase a la que pertenece $x$ (por ejemplo, la clase de los jardineros, de los corazones, de los sacacorchos, etc.) y, después, determinamos la calidad con que $x$ realiza $\varphi$. Evaluamos a $x$ como un buen $x$ si realiza buenas instancias de $\varphi$; por ejemplo, evaluamos un corazón como un buen corazón si bombea bien la sangre, pues ésta es la función característica o el ergon de los miembros de la clase de los corazones.

Aristóteles, efectivamente, reconoce el ergon o la función peculiar de una cosa como aquello que hay que considerar al evaluarla cuando dice, por ejemplo, "para el flautista y para el escultor y para todo artesano, y en general para todos aquellos que producen obras o que desempeñan una actividad, en la obra que realizan se cree que residen el bien y la perfección". ${ }^{17}$ Sin embargo, él también hace hincapié en que no es propiamente el ergon lo que distingue a un buen $x$ de, digamos, un mal $x$ o un mediocre $x$, puesto que todos tienen un mismo ergon y es precisamente por eso que pertenecen a la misma clase de cosas; lo distintivo de un buen $x$ es la excelencia con que ejecuta su ergon; ${ }^{18}$ por ejemplo, lo que distingue a un buen médico de uno malo es la excelencia de su práctica médica.

Ahora bien, es evidente que en el argumento de Gómez-Lobo no se da un paso inválido del es al debe para llegar a la conclusión 2(iii) porque, según hemos visto, la premisa 2(i) es también evaluativa y, junto con la premisa fáctica 2(ii), permite inferir válidamente 2(iii). Si el argumento es una reconstrucción correcta de lo que sostiene Aristóteles, no cabe objetar, afirma Gómez-Lobo, que en la defensa de su concepción de la eudaimonia se comete la falacia naturalista. ${ }^{19}$ Pero ¿acaso tiene razón Gómez-Lobo en esto último?

El trabajo de interpretación textual y de análisis de la $E N$ que lleva a cabo Gómez-Lobo parece impecable. Sin embargo, para descartar la crítica de que la concepción aristotélica de la eudaimonia comete la falacia naturalista - lo cual es el asunto en discusión - no basta con mostrar la validez lógica de dicho argumento, sino que se requiere, además, mostrar que tampoco se comete la falacia naturalista al defender cada una de las premisas que lo componen. Así que cabe preguntar: cla defensa de la premisa 2(i) no supone acaso un paso injustificado del es al debe? Recordemos que 2(i) establece que para cualquier $x$ que tenga un ergon $\varphi, x$ será un buen $x$ si y sólo si produce buenas instancias de $\varphi$; por

${ }^{17}$ Aristóteles, EN 1097b25-30.

${ }^{18}$ Cfr. ibid., 1098a5-15.

${ }^{19}$ Cfr. Gómez-Lobo 1989, pp. 170 y 182; Gómez-Lobo 1991, pp. 2-3 y 13. 
ejemplo, ya que el ergon o la función propia de los serruchos es cortar madera, un serrucho particular será un buen serrucho si y sólo si corta bien la madera. ¿Es ésta una tesis aceptable?

Alguien podría señalar que, independientemente de que sea o no aceptable, 2(i) es irrelevante como parte de un argumento sobre la eudaimonia, porque - a diferencia de "serrucho"- "ser humano" no es un término funcional, es decir, un término que se refiere a una cosa que se distingue de las demás por la función que realiza o por aquello para lo que sirve. ${ }^{20}$ A esta crítica se podría responder que Aristóteles parece identificar la "función propia" o ergon de una cosa con su "forma" o eidos y no con su función o aquello para lo que sirve; en efecto, tanto la función propia como la forma de una cosa son caracterizadas en algunos pasajes de su obra como aquello que hace que la cosa sea lo que es, como aquello que la define, ${ }^{21}$ de modo que preguntarse cuál es la función propia de los seres humanos es lo mismo que preguntarse cuál es su forma, esto es, qué es lo que hace que los humanos sean la clase de cosas que son. Pero, prescindiendo de las críticas que hoy en día se puedan hacer a las nociones aristotélicas de función propia y forma, así como al papel de éstas en la concepción de lo que sea una buena vida para los humanos, se puede objetar a la reconstrucción del argumento sobre la eudaimonia que ofrece Gómez-Lobo que 2(i) es una premisa evaluativa que se infiere por inducción enumerativa a partir de premisas fácticas, con lo cual claramente se comete la falacia naturalista. Examinemos esto último con mayor detalle.

Hemos visto que Gómez-Lobo sostiene que para Aristóteles 2(i) parece ser algo así como "un principio evaluativo de sentido común obtenido por una inducción enumerativa". ${ }^{22}$ En apoyo de esta manera de considerar a 2(i) Gómez-Lobo afirma:

${ }^{20}$ John Cooper y J.O. Urmson están entre quienes argumentan que atribuirles a los humanos un ergon supone cometer el error de considerar a "ser humano" un término funcional (cfr. Cooper 1986, pp. 146-147; Urmson 1988, p. 19). "Serrucho", "corazón" o "médico", por ejemplo, son términos funcionales, pero no lo son "ser humano", "caballo" o "aguacate", porque las cosas a las que estos últimos términos refieren no tienen por naturaleza una función o un uso que las defina como lo que son. Es verdad que la gente suele usar a los caballos para cargar cosas, trasladarse de un lugar a otro o tirar carruajes, pero, dice Urmson, "esto muestra solamente que el hombre es el imperialista del reino animal y no que los caballos, por su propia naturaleza, tengan una función que realizar" (Urmson 1988, p. 19; la traducción es mía).

${ }^{21}$ Cfr. Aristóteles, Meteorologica, 390a10-13; Aristóteles, Metafísica, 1032b1 y 1035b15-25.

${ }^{22}$ Véase la nota 16.

Diánoia, vol. LV, no. 65 (Noviembre 2010). 
En efecto, yo diría que normalmente conducimos nuestras evaluaciones en esta forma. Identificamos lo que es específico de la clase a la cual pertenece un individuo (la clase de los arquitectos, los hoteles, las azaleas, los bulldogs, etc.) y luego procedemos a preguntar si éste en particular representa bien aquellas actividades o características. ${ }^{23}$

Por una generalización de lo que de hecho hacemos cuando emitimos juicios evaluativos acerca de un arquitecto, una azalea, un hotel, un bulldog, etc., supuestamente inferimos de manera inductiva el principio evaluativo 2(i). ${ }^{24}$ Pero si éste fuera el caso, ¿no es obvio que estaríamos dando un paso injustificado del es al debe? Hasta donde puedo ver, de manera inductiva lo único que podemos inferir válidamente a partir de las premisas fácticas en cuestión es una conclusión también fáctica sobre cómo de hecho hacemos evaluaciones; la inferencia puede presentarse así:

\section{ARgumENTO 3}

(i) Evaluamos a a como un buen arquitecto si produce buenas instancias del ergon de los arquitectos, esto es, de la actividad o las características que lo hacen pertenecer a la clase de los arquitectos.

(ii) Evaluamos a $b$ como un buen bulldog si produce buenas instancias del ergon de los bulldogs, esto es, de la actividad o las características que lo hacen pertenecer a la clase de los bulldogs.

Y así sucesivamente para todos los individuos que evaluamos.

Por lo tanto,

(iii) Para todo $x$, si $x$ tiene un ergon $\varphi$, evaluamos a $x$ como un buen $x$ si produce buenas instancias de $\varphi$.

La conclusión 3(iii) describe el procedimiento que de hecho seguimos las personas para hacer juicios del tipo "este $x$ es un buen $x$ ", mientras que 2(i) en la reconstrucción del argumento aristotélico de GómezLobo es un juicio evaluativo acerca de lo que hace que algo sea un

${ }^{23}$ Gómez-Lobo 1989, p. 183. La traducción de ésta y las subsiguientes citas del texto de Gómez-Lobo es mía.

${ }^{24}$ Para Aristóteles, la premisa 2(i) es, cito textualmente a Gómez-Lobo, "un principio obtenido por una sumaria inducción [sic]" (Gómez-Lobo 1991, p. 6). El concepto de "sumaria inducción" no existe; a lo que Gómez-Lobo pretende referirse en la cita anterior es a una inducción enumerativa, es decir, a una generalización que se confirma por la observación de casos particulares. 
buen $x$. 3(iii) enuncia simplemente las condiciones en las que es correcto aseverar que $x$ es un buen $x$, en tanto que 2(i) dice qué requiere un $x$ para ser un buen $x$. Una y otra cosa son obviamente distintas $y$, en principio, podría satisfacerse una y no la otra; por ejemplo, podría ser correcto aseverar que alguien es inocente porque no se cuenta con elementos de prueba suficientes para culparlo, sin que fuera verdad que es inocente. Pero lo relevante para lo que aquí se discute es que 3(iii) y no 2(i) es lo que es válido inferir inductivamente a partir de 3(i), 3(ii), etcétera. Considerar que 2(i) está justificada por inducción enumerativa a partir de premisas como 3(i), 3(ii), etc. -lo que hace Aristóteles, según Gómez-Lobo- permite que se plantee nuevamente la acusación de cometer la falacia naturalista. En este sentido, la defensa que ofrece Gómez-Lobo no parece lograr su cometido.

En las siguientes dos secciones examinaré una manera distinta (y a mi juicio más prometedora que la de Gómez-Lobo) de demostrar que es posible defender una concepción aristotélica de la eudaimonia sin cometer la falacia naturalista.

\section{Evaluaciones de bondad relativas a seres vivos. Una perspectiva neoaristotélica}

Se pueden hacer evaluaciones muy diversas acerca de los seres vivos. Por ejemplo, evaluamos una característica física cuando decimos que el naranjo de nuestro jardín tiene buenas raíces, evaluamos una facultad mental cuando juzgamos que nuestro perro tiene mala memoria y evaluamos una intención y una acción, respectivamente, cuando decimos que nuestras intenciones eran buenas al ocultarle la verdad a nuestra madre enferma o que uno de nuestros colegas actuó mal en determinada situación. ¿Cómo se justifican evaluaciones como las anteriores? ¿Con respecto a qué cabe calificar como buenas o malas las características, facultades, acciones e intenciones de los individuos?

Evaluar como buenos o malos los rasgos de un determinado animal o planta sólo parece tener sentido si se tiene como marco de referencia las descripciones empíricas (como las que proporcionan la botánica, la anatomía, la etología, la sociobiología, etc.) acerca de la especie a la que pertenece el individuo en cuestión. Así, por ejemplo, no parece tener sentido decir que el colibrí que viene a mi jardín todas las mañanas tiene el pico defectuoso, si no es con respecto a las descripciones hechas por la anatomía animal acerca del pico de los pájaros de dicha especie; el hecho de que el colibrí que viene a mi jardín no tenga el pico tan fuerte como el de un pájaro carpintero o tan grueso como el de los 
pájaros de otras especies no puede ser considerado en sí mismo un defecto. De manera similar, no parece tener sentido decir que algo anda mal con el modo en que mi gata trata a sus crías, si no es con respecto a las descripciones hechas por la etología acerca de la forma de crianza característica de los miembros de la especie a la que pertenece mi gata. Según lo anterior, evaluar como defectuoso un rasgo particular de un ser vivo equivale a señalar que dicho rasgo se aparta de lo que es propio de la especie a la que pertenece.

Pero ¿̇cualquier característica o comportamiento de un individuo que se salga de lo que es la norma para su especie tiene que considerarse una deficiencia? Por ejemplo, ¿es un defecto en mi perro que tenga una pequeña mancha café en una de sus patas, porque los perros de su especie suelen tener todo el pelo blanco?, ¿es acaso una deficiencia en él, como lo sería el que careciera de colmillos o el que tuviera sólo tres patas? La respuesta a estas preguntas es "no", pues sólo cabe considerar como deficiencia una desviación de un individuo respecto de cierto rasgo de su especie en cuanto que, como consecuencia de esa desviación, se vea afectada alguna función en la vida de ese individuo - por ejemplo, alguna función relativa a su desarrollo, su alimentación, su capacidad para defenderse, su reproducción, y, en general, cualquiera otra función relacionada con lo que los zoólogos calificarían como su forma de vida.

De acuerdo con lo anterior, para que podamos concluir que algo es un defecto en un individuo tenemos que contar con un argumento que tenga la forma siguiente:

\section{ARGUMENTO 4}

(i) Una premisa mayor que describa algún rasgo de los miembros de la especie en cuestión, el cual hace posible que realicen alguna función relacionada con su forma de vida.

(ii) Una premisa menor que diga si un determinado individuo de la especie en cuestión posee o no posee el rasgo descrito en la premisa mayor.

(iii) Una conclusión en la que se evalúe si el individuo del que se habla en la premisa menor es como debería de ser en relación con el rasgo al que alude la premisa mayor o si, por el contrario, tiene alguna deficiencia relacionada con él.

Neoaristotélicos como Alasdair MacIntyre y Philippa Foot, al igual que algunos otros filósofos que han defendido concepciones naturalistas de 
lo bueno, como Michael Thompson y Paul Taylor, coinciden en señalar que las evaluaciones sobre seres vivos individuales se justifican observando cómo son y comparándolos con las descripciones empíricas de la especie a la que pertenecen. ${ }^{25}$ Para que esas evaluaciones atribuyan justificadamente alguna deficiencia a un ser vivo es necesario que se basen en la detección de alguna desviación del individuo respecto de su especie, la cual afecte de manera directa o indirecta alguna función relativa a su forma de vida. Si esto es correcto, a primera vista no cabe acusar a las éticas aristotélicas de cometer la falacia naturalista al justificar evaluaciones sobre la bondad de determinados propósitos, acciones, intenciones, etc., a partir de descripciones sobre la forma de vida de los seres humanos, de la misma manera en que no cabe acusar a un zoólogo de cometer dicha falacia al justificar evaluaciones acerca de qué tan bien se comunica un delfín con su manada a partir de descripciones sobre la forma de vida propia de los individuos de su especie.

Pero si no se comete una falacia al justificar una conclusión evaluativa del tipo 4(iii) a partir de premisas fácticas del tipo 4(i) y 4(ii), entonces, la relación entre una y otras no puede ser de implicación lógica. ¿Qué relación hay entre la conclusión y las premisas? En la siguiente sección intentaré responder a esta pregunta, pero antes conviene hacer algunas precisiones en relación con los juicios acerca de la vida buena para un ser vivo.

En contraste con las evaluaciones sobre rasgos específicos de un individuo, como sus pulmones, su memoria, sus intenciones al realizar una determinada acción, etc., juzgar que un individuo tiene una vida buena supone hacer una valoración global de su condición. Así, por ejemplo, evaluar como buena o eudaímōn la vida de una persona supone valorar los diversos aspectos que conforman su vida y para que esa evaluación esté justificada, como dice Jean Austin, "nada esencial debe estar mal". ${ }^{26}$ Dado que un juicio acerca de la bondad de la vida de un individuo es un juicio global de su condición y dado que, como hemos dicho, en el caso de cualquier ser vivo las evaluaciones de bondad sólo tienen sentido por referencia a alguna función relativa a la forma de vida de su especie, entonces, hacer un juicio sobre la bondad de la vida de un individuo sólo tiene sentido por referencia al conjunto de las funciones que constituyen la forma de vida de su especie.

El conjunto de descripciones acerca de cómo los individuos de una determinada especie logran los objetivos relativos a su forma de vida

${ }^{25}$ Cfr. Foot 2001, pp. 5-37; MacIntyre 2001, pp. 10, 81-97; Thompson 1995, pp. 281-295; Thompson 2003; Taylor 1998, pp. 277-280; Taylor 1986, p. 60.

${ }^{26}$ Austin 1968, p. 238; la traducción es mía. 
permite establecer un conjunto de estándares o de normas, tanto a nivel individual como social, para los miembros pertenecientes a esa especie. ${ }^{27}$ Por ejemplo, para que los lobos puedan realizar las funciones propias de su ciclo vital deben o necesitan tener colmillos de cierto tipo, patas con determinadas características, cazar en grupo, etcétera. La aplicación de este conjunto de normas o de estándares a un miembro individual de la especie es lo que permite juzgar si ese individuo es o no como debería ser para poder dar cumplimiento a la forma de vida propia de su especie o, dicho de otro modo, lo que permite evaluar si ese individuo posee o no posee bondad natural. La estructura conceptual de estas "evaluaciones sobre la bondad natural de los seres vivos", como les llama Foot, ${ }^{28}$ permite distinguirlas de otros tipos de evaluaciones, como las instrumentalistas, las estéticas, etcétera. Un tigre o un olivo, por ejemplo, pueden valorarse desde el punto de vista de sus propiedades nutritivas para los humanos y ésas son evaluaciones de tipo instrumental; también pueden valorarse aspectos como la armonía y la belleza de sus formas y ésas son evaluaciones de tipo estético; ya que ninguna de estas evaluaciones se justifica por referencia al cumplimiento de la forma de vida de los tigres o de los olivos, no son evaluaciones de su bondad natural.

La bondad natural es una condición necesaria para que el individuo logre tener una vida buena $-o$ dicho de otra forma, para que pueda florecer qua miembro de su especie o realizar plenamente su bien propio; sin embargo, no parece ser una condición suficiente, pues algunos factores ambientales o externos al individuo pueden entorpecer e, incluso, impedir dicho logro. ${ }^{29}$ Los factores externos que pueden entorpecer la realización de una vida buena son de dos tipos diferentes: por un lado, factores que interfieren directamente con las funciones naturales de desarrollo, alimentación, reproducción, etc.; por ejemplo, sufrir un grave accidente, vivir en la miseria o en un ambiente sumamente contaminado, etcétera. Por otro lado, factores que no interfieren directamente con las funciones naturales - como sería el caso de la muerte o la enfermedad grave de un ser amado, la pérdida de la fortuna (sin

${ }^{27}$ Cfr. lo que dicen a este respecto Foot 2001, pp. 25-37 y 91-92; MacIntyre 2001, pp. 82-83; Thompson 1995, pp. 295-296; Thompson 2003; Taylor 1986, pp. 6667.

${ }^{28}$ Cfr. Foot 2001, p. 3.

${ }^{29}$ Acerca de la importancia que los neoaristotélicos y el propio Aristóteles conceden a las condiciones externas o ambientales en el logro de una vida buena, véanse: Foot 2001, pp. 15, 31, 41-42; MacIntyre 2001, pp. 82-83; Aristóteles, EN 1099a24-1099b9. 
quedar en la miseria), etc.- pero que pueden producir, por ejemplo, depresión o tristeza, lo cual sí dificulta la realización de algunas de dichas funciones.

Considerando lo dicho en esta sección, ¿cuál sería la estructura de un argumento neoaristotélico sobre la eudaimonia o la vida buena para un ser humano? En lo que sigue intentaré contestar a esta pregunta, además de ofrecer una explicación de la relación que hay entre las premisas y la conclusión de un argumento tal que no dé lugar a que se plantee la crítica de un tránsito injustificado del es al debe.

\section{Una concepción neoaristotélica, no falaz, de la vida buena}

Asumiendo una perspectiva neoaristotélica, como la presentada en la sección anterior, cabe sostener que para concluir que un ser humano tiene una vida buena se requiere contar con un argumento de la forma siguiente:

\section{ARGUMENTO 5}

(i) El conjunto de funciones $\varphi^{1}, \varphi^{2}, \varphi^{3} \ldots \varphi^{n}$ constituye la forma de vida propia de la especie humana.

(ii) La persona $A$ ha logrado (o no) las funciones $\varphi^{1}, \varphi^{2}, \varphi^{3} \ldots \varphi^{n}$. Por lo tanto,

(iii) La persona $A$ tiene (o no) una vida buena.

Las dos premisas del argumento anterior son fácticas. Una premisa como 5(i) puede justificarse, en principio, a partir de la información que proporcionan ciencias empíricas como la anatomía, la neurología, la sociología, la psicología, la antropología, etc.; la información que estas ciencias proporcionan puede permitir, por ejemplo, caracterizar la forma de vida de los humanos a partir de funciones tales como lograr tener salud corporal, ser capaz de imaginar y razonar, comprometerse en formas diversas de interacción social, tener amigos, tener posibilidad de cultivar algunos de los propios intereses, participar en la educación, etcétera. ${ }^{30}$ La importancia de una premisa como 5(i) para las concep-

\footnotetext{
${ }^{30}$ Martha Nussbaum ha propuesto una lista de capacidades para realizar funciones que son de importancia central para la forma de vida humana; esas capacidades se refieren a los siguientes diez aspectos: vida; salud corporal; integridad corporal; sentidos, imaginación y pensamiento; emociones; razón práctica; afiliación; relación con otras especies; juego; y control del propio entorno. Cfr. Nussbaum 2002, pp. 110-123.
}

Diánoia, vol. LV, no. 65 (Noviembre 2010). 
ciones neoaristotélicas de la vida buena es evidente en las propuestas de Foot (2001) y MacIntyre (2001). En sus respectivos análisis de las funciones constitutivas de la forma de vida humana ambos examinan el papel que en la realización de tales funciones tienen las diversas características y capacidades físicas y mentales de los individuos de nuestra especie, tanto las que compartimos con otros animales (por ejemplo, al igual que otros primates tenemos manos prensiles, la capacidad de ver, reconocer objetos, etc.), como las que son exclusivas nuestras (por ejemplo, un determinado tipo de laringe, un cierto tipo de aparato auditivo o la imaginación).

Pero, más allá de la consideración de la manera en la que intervienen diversas características y capacidades físicas y mentales de los humanos en la realización de las funciones propias de la forma de vida de su especie, Foot y MacIntyre enfatizan el papel especial que tiene la racionalidad humana. ${ }^{31} \mathrm{Si}$ hay algo que es propio de la vida de los humanos, de acuerdo con el naturalismo aristotélico, es precisamente el ser capaces de actuar intencionalmente, es decir, de realizar las diversas funciones que integran nuestra forma de vida basándonos en razones, ser capaces de guiarnos por razones que consideramos al plantearnos distintos propósitos acerca de los diversos aspectos de nuestra vida y al actuar para intentar conseguirlos. A otros animales distintos de los humanos tal vez quepa atribuirles alguna capacidad similar a la razón práctica, ${ }^{32}$ pero lo cierto es que sólo los humanos somos capaces de actuar basándonos en razones que formulamos proposicionalmente y que conectamos lógica y semánticamente con otras proposiciones. Más aún, los humanos somos capaces de reflexionar acerca de las razones que tenemos para realizar una determinada acción, evaluando con diversos criterios nuestros propios juicios al respecto.

Sobre la premisa 5(ii) del argumento no parece necesario dar mayores explicaciones. Es claro que al igual que 5(i) es una premisa empírica y que para justificarla se requiere contar con información acerca de los

\footnotetext{
${ }^{31}$ Véanse, por ejemplo, Foot 2001, pp. 52-65; MacIntyre 2001, pp. 71-79, 99117.

${ }^{32}$ MacIntyre, por ejemplo, sostiene que cabe reconocerles la capacidad de la razón práctica a algunos animales como los chimpancés o los delfines, en tanto que son capaces de percibir determinadas conexiones entre los bienes que desean y los medios que permiten alcanzarlos y eso les da una razón para actuar como lo hacen. Éstas son razones que MacIntyre califica de "prelingüísticas", aunque, atendiendo a la distinción que hace José Luis Bermúdez entre criaturas lingüísticas y no lingüísticas, tal vez sea más adecuado calificarlas como razones "no lingüísticas" (cfr. MacIntyre 2001, pp. 53-59, 74-78; Bermúdez 1998, p. 46).
} 
diversos aspectos de la vida de la persona en cuestión. Esta información es la que permite establecer si ella realiza o no el conjunto de funciones descritas en 5(i), por ejemplo, si participa de los procesos educativos o se ve excluida de ellos, si puede cultivar su gusto por la jardinería o se lo impide una jornada laboral agobiante, si participa en alguna medida en la vida política de su comunidad o no, etcétera.

En contraste con 5(i) y 5(ii), la conclusión 5(iii) del argumento es evaluativa, pues no describe qué funciones de hecho realiza una determinada persona $A$, sino que evalúa si su vida considerada como un todo es o no buena. Evidentemente, el adjetivo "buena" que aparece en 5(iii) no se explica por referencia a la satisfacción de cualesquiera que sean los deseos que tenga $A$; o, más precisamente, satisfacer determinados tipos de deseo contribuirá a la bondad de la vida de $A$ sólo en la medida en que eso sea una de las funciones propias de la forma de vida de nuestra especie. Sostener la conclusión 5(iii), pues, supone rechazar la tesis compartida por emotivistas y prescriptivistas según la cual toda evaluación acerca de "lo bueno" se explica necesariamente por referencia exclusiva a los deseos, intereses o preferencias de quienes hacen tal afirmación, esto es, se explica únicamente con base en consideraciones subjetivas.

¿Cómo es que 5(i) y 5(ii) permiten justificar 5(iii)? De acuerdo con lo dicho en la sección anterior, las evaluaciones de "bondad" en el caso de seres vivos individuales se justifican por referencia a la realización de las funciones que constituyen la forma de vida de la especie a la que pertenece el individuo en cuestión. Y lo que es cierto para individuos de otras especies animales lo es también para los individuos de la especie humana. Dado que evaluar como buena la vida de un individuo supone hacer una valoración global de su condición, y dado que la noción de "forma de vida de la especie $E$ " es omniabarcante respecto de las funciones propias del ciclo vital de los individuos que pertenecen a $E$, se justifica concluir que una persona $A$ tiene una vida buena, como se hace en 5(iii), una vez que se ha establecido que la especie a la que pertenece $A$ tiene una determinada forma de vida y que $A$ ha logrado el conjunto de las funciones que constituyen la forma de vida de su especie. Notemos, además, que es precisamente porque se requiere el logro del conjunto de estas funciones - y no solamente de una de ellasque cabe considerar como inclusivista esta concepción de la vida buena.

Pero ¿no se está pasando por alto algún aspecto del argumento 5? ¿No hay entre las premisas algún concepto evaluativo, pese a que se pretende que son premisas fácticas? Alguien tal vez podría sostener que en efecto 5(i) es evaluativa, porque en ella se habla de la "forma de vida 
do la verdad o falsedad de $D$ se justifica con base en $H .{ }^{34}$ Por ejemplo, en el contexto de nuestras instituciones del dinero y de la compraventa, explica ella, los hechos $x$ le pidió a z papas, él se las mandó y le envió una factura que $x$ no ha pagado son hechos brutos relativamente a la descripción " $x$ le debe a $z$ tal cantidad", porque esta descripción es verdadera en virtud de aquellos hechos; a su vez, el hecho $x$ le debe $a z$ tal cantidad es un hecho bruto relativamente a la descripción " $x$ es deudor". ${ }^{35}$

De manera similar al caso anterior, el hecho La persona A logra (o no) realizar las funciones $\varphi^{1}, \varphi^{2}, \varphi^{3} \ldots \varphi^{n}$, al que llamaré $H_{f}$, puede considerarse un hecho bruto relativamente a la descripción $D H_{e}$ " $A$ realiza (o no) la forma de vida propia de su especie", porque el valor de verdad de esta descripción se determina con base en aquel hecho. Ahora bien, en el inciso a) anterior he dicho que el significado de 5(i) no depende de la noción de "bien" ni de cualquiera otra noción evaluativa; considerando a $H_{f}$ como un hecho bruto relativamente a $D H_{e}$ podríamos decir que para entender el significado de 5(i) basta con entender la relación entre $\mathrm{DH}_{e}$ y $\mathrm{H}_{f}$.

Así como $H_{f}$ es un hecho bruto relativamente a la descripción $D H_{e}$, el hecho $H_{e}$ descrito por ésta, es decir La persona $A$ ha logrado (o no) realizar la forma de vida propia de la especie humana, es un hecho bruto relativamente a la descripción $D H_{b}$ " $A$ tiene (o no) una vida buena". Como podemos constatar, $D H_{b}$ sí incluye una noción evaluativa: la noción de "vida buena". $H_{e}$ es un hecho bruto relativamente a $D H_{b}$ porque, en el contexto de las evaluaciones de bondad relativa a seres vivos, el valor de verdad de dicha descripción se determina por tal hecho.

En 5(iii) se concluye, precisamente, que $D H_{b}$ es el caso y dicha conclusión se justifica a partir de premisas acerca de las funciones que constituyen la forma de vida humana y de la realización de tales funciones por parte de la persona $A$, pero, por supuesto, la relación entre la conclusión y las premisas no es de implicación lógica — por ello no cabe la acusación de que se comete la falacia naturalista. Es en el contexto de las evaluaciones de bondad relativa a seres vivos y de su particular estructura conceptual (a las que hemos visto que Foot llama "evaluaciones sobre la bondad natural de los seres vivos") ${ }^{36}$ donde se justifica concluir 5(iii) a partir de 5(i) y 5(ii); lo cual equivale a sostener que en ese contexto los hechos aseverados por estas premisas son "hechos brutos" relativamente a la conclusión 5(iii).

${ }^{34}$ Cfr. Anscombe 1958b, p. 71.

${ }^{35} \mathrm{Cfr}$. Anscombe 1958a, pp. 3-4.

${ }^{36}$ Véase la n. 28. 
Las evaluaciones sobre bondad natural son distintas, evidentemente, de otras evaluaciones que pueden también justificarse adecuadamente de acuerdo con el contexto que les es propio; por ejemplo, evaluaciones instrumentalistas, como "La manzanilla es buena para aliviar el cólico", evaluaciones sobre satisfacción de preferencias, como "Fue bueno para mí tener hijos y mudarme de ciudad", etc. La relevancia de una concepción neoaristotélica de la vida buena como la delineada en este artículo reside en gran parte, creo yo, en defender exitosamente la posibilidad de ofrecer una evaluación genuina y no falaz sobre la bondad de la vida humana, que no se justifica por mera referencia a la satisfacción de deseos, preferencias o gustos personales, sino por referencia a lo que objetivamente puede ser considerado bueno para un individuo de la especie humana.

\section{Conclusión}

He intentado mostrar que es posible defender una concepción de la vida buena de corte aristotélico y de tipo inclusivista sin dar un paso injustificado del es al debe. Esta concepción recurre, por un lado, a un análisis de la justificación de cualquier evaluación de bondad relativa a seres vivos y, por otro lado, al concepto de "ser un hecho bruto relativamente a..." introducido por G.E. Anscombe. Teniendo en cuenta estos dos elementos teóricos he defendido que es posible justificar el valor de verdad de una conclusión acerca del logro de una vida buena por parte de una persona, a partir de premisas fácticas acerca de las funciones que constituyen la forma de vida humana y la realización (o no) de tales funciones por parte de esa persona; los hechos aseverados por tales premisas son hechos brutos relativamente al enunciado que concluye que la persona tiene (o no) una vida buena.

Por supuesto, hay muchas cuestiones aún por aclarar para hacer viable una concepción de corte aristotélico sobre la vida buena como la que he intentado defender en este artículo. Una especialmente importante es si realmente podemos contar con una caracterización satisfactoria de lo que significa para un individuo humano realizar la forma de vida propia de su especie. Mientras más compleja es una especie, se torna más difícil poder decir en qué consiste para un individuo perteneciente a ella dar cumplimiento a su forma de vida, porque, para eso, se requiere contar con una caracterización completa, coherente y, por supuesto, verdadera de la forma en que los miembros de esa especie realizan las funciones propias de su ciclo vital. 
Además, es preciso tener en cuenta que aunque es cierto que hay características que todos los humanos compartimos con independencia del momento sociohistórico y del lugar donde vivamos (de otro modo no tendría sentido hablar de "la especie humana"), hay también variaciones importantes a través del tiempo y del espacio en la manera peculiar que distintos grupos humanos tienen de realizar diversas funciones propias de la vida humana e, incluso, en la manera en que lo hacen distintos individuos dentro de un mismo grupo humano. Una concepción aristotélica de la vida buena tiene que dar cabida a la diversidad de maneras de ejemplificar la forma de vida propia de la especie humana.

Hay varias cuestiones, pues, que requieren ser examinadas, pero espero haber mostrado que, en principio, es posible defender una concepción de la vida buena de corte aristotélico sin dar un paso injustificado del es al debe.*

\section{BIBLIOGRAFÍA}

Ackrill, J.L., 1980, "Aristotle on Eudaimonia", en Amélie Oksenberg Rorty (comp.), Essays on Aristotle's Ethics, University of California Press, Berkeley/Los Ángeles, pp. 15-33.

Annas, Julia, 1996, "Aristotle and Kant on Morality and Practical Reasoning", en Stephen Engstrom y Jennifer Whiting (comps.), Aristotle, Kant and the Stoics. Rethinking Happiness and Duty, Cambridge University Press, Cambridge, pp. 237-257.

Anscombe, G.E.M., 1958a, "Modern Moral Philosophy", Philosophy, vol. 33, no. 124, pp. 1-19.

— 1958 b, "On Brute Facts", Analysis, vol. 18, no. 3, pp. 69-72.

Aristóteles, 1998, Metafísica, trad. Tomás Calvo Martínez, Gredos, Madrid (Biblioteca Clásica, 200).

—_, 1983, Ética Nicomáquea, 2a. ed., trad. Antonio Gómez Robledo, Instituto de Investigaciones Filológicas-UNAM, México.

—, 1952 , Meteorologica, trad. H.D.P. Lee, Harvard University Press, Cambridge, Mass.

Austin, Jean, 1968, "Pleasure and Happiness", en J.B. Schneewind (comp.), Mill: A Collection of Critical Essays, Anchor Books, Nueva York, pp. 234-250.

Bermúdez, José Luis, 1998, The Paradox of Self-Consciousness, Massachusetts Institute of Technology Press, Cambridge, Mass.

Cooper, John, 1986, Reason and Human Good in Aristotle, Hackett Publishing Company, Indianápolis.

*Agradezco mucho a Margarita Valdés sus valiosas observaciones y sugerencias tras su lectura de algunas versiones anteriores de este artículo; también deseo agradecer a los dictaminadores anónimos de Diánoia sus útiles comentarios.

Diánoia, vol. LV, no. 65 (Noviembre 2010). 
Foot, Philippa, 2001, Natural Goodness, Clarendon Press, Oxford. [Versión en castellano: Bondad natural. Una visión naturalista de la ética, trad. Ramón Vilà Vernis, Paidós, Barcelona, 2002.]

Gómez-Lobo, Alfonso, 1991, "La fundamentación de la ética aristotélica", Diánoia, no. 37, pp. 1-15.

—, 1989, "The Ergon Inference", Phronesis, vol. 34, pp. 170-184.

Gómez Robledo, Antonio, 1957, Ensayo sobre las virtudes intelectuales, Fondo de Cultura Económica/Centro de Estudios Filosóficos-UnAM, México.

Hume, David, 1978, A Treatise of Human Nature, ed. L.A. Selby-Bigge, 2a. ed., Clarendon Press, Oxford.

Hunter, Geoffrey, 1969, "Hume on Is and Ought", en W.D. Hudson (comp.), The Is-Ought Question. A Collection of Papers on the Central Problem in Moral Philosophy, MacMillan, Londres, pp. 59-63.

Irwin, T.H., 1993, "La concepción estoica y la concepción aristotélica de la felicidad", en Malcolm Schofield y Gisela Striker (comps.), Las normas de la naturaleza. Estudios de ética helenística, trad. Julieta Fombona, Manantial, Buenos Aires, pp. 211-250.

Kenny, Anthony, 1965-1966, "Happiness", Proceedings of the Aristotelian Society, vol. 66, pp. 87-102.

Lawrence, Gavin, 1997, "Nonaggregatability, Inclusiveness, and the Theory of Focal Value: Nicomachean Ethics 1.7.1097b16-20", Phronesis, vol. 42, no. 1, pp. 32-76.

MacIntyre, Alasdair, 2001, Animales racionales y dependientes. Por qué los seres humanos necesitamos las virtudes, trad. Beatriz Martínez de Murguía, Paidós, Barcelona. [Traducción de: Dependent Rational Animals. Why Human Beings Need the Virtues, Open Court, Chicago, 1999.]

_ 1969 , "Hume on 'Is' and 'Ought'”, en W.D. Hudson (comp.), The Is-Ought Question. A Collection of Papers on the Central Problem in Moral Philosophy, MacMillan, Londres, pp. 35-50.

Nussbaum, Martha C., 2002, Las mujeres y el desarrollo humano, trad. Roberto Bernet, Herder, Barcelona.

Rorty, Amélie O., 1980, "The Place of Contemplation in Aristotle's Nicomachean Ethics", en Amélie Oksenberg Rorty (comp.), Essays on Aristotle's Ethics, University of California Press, Berkeley/Los Ángeles, pp. 377-394.

Taylor, Paul W., 1998, "La ética del respeto a la naturaleza", en Teresa Kwiatkowska y Jorge Issa (comps.), Los caminos de la ética ambiental. Una antología de textos contemporáneos, trad. Jorge Issa, CONACYT/UAM-I/Plaza y Valdés, México, pp. 269-287.

- 1986, Respect for Nature. A Theory of Environmental Ethics, Princeton University Press, Princeton.

Thompson, Michael, 2003, "Three Degrees of Natural Goodness", disponible en $<$ http://www.pitt.edu/ ${ }^{m}$ thompso/three.pdf $>$, consultado el 3 de diciembre de 2008. 
Thompson, Michael, 1995, "The Representation of Life", en R. Hursthouse, G. Lawrence y W. Quinn (comps.), Virtues and Reasons. Philippa Foot and Moral Theory, Oxford University Press, Oxford, pp. 247-296.

Urmson, J.O., 1988, Aristotle's Ethics, Basil Blackwell, Oxford.

Valdés, Margarita M., 1991, "Dos aspectos en el concepto de bienestar", Doxa, no. 9, pp. 69-89.

Recibido el 7 de noviembre de 2009; aceptado el 10 de agosto de 2010. 